\title{
Needs assessment for the management of fecal incontinence
}

\author{
I. Raîche, R. Helewa, L.Williams,, K. Foss, W. Baksh-Thomas, H. Moloo
}

\section{Introduction:}

- A large proportion of Canadians suffer with fecal incontinence

- Conservative measures such as dietary modification, pelvic floor physiotherapy, and biofeedback are effective in $50 \%$ of patients

- Before implementing a new program to improve our care to patients with fecal incontinence, we decided to conduct a need assessment.

- The goals of this exploratory study were:

- To identify what would constitute success when treating patients with fecal incontinence both from a patient and a healthcare provider's perspective

- To identify barriers and promoters that could impact a fecal incontinence program's sustainability.

\section{Methods:}

- This was a cross-sectional, qualitative study

- Standardized, semi-structured interviews and focus groups were conducted

- The participants:

- Purposeful sample

- Patients suffering from fecal incontinence, nurses, physical therapists, and physicians who treat fecal incontinence

- Interview questions revolved around success definitions, barfiers and promoters of fecal incontinence care

- Transcripts were analyzed using content analysis methodology to develop themes surrounding fecal incontinence care

- Saturation was achieved, i.e. no new themes emerged in the last two transcripts analyzed

- Two analysts independently coded the transcripts with the final coding system and achieved an inter-rater reliability kappa of 0.86

Results:

- Twelve interviews were undertaken with 16 participants

- 17 different themes were identified .

- Barriers to fecal incontinence care :

- Education for both the care provider and patients.

"I just thought, is this another thing with aging? You know."

- Access issues for fecal incontinence treatments were also highlighted.

"right now, there's not... there's maybe some conservative measures and, once those are exhausted, there's no other treatment for patients to turn to."

\section{- Promoters of fecal incontinence care}

- Impact that FI has on quality of life (personal hygiene, psychological burden, and activity and productivity)

"Well, it slows me down. I've... I'm finding I'm limiting where I go and what I do a little bit, not a lot, but I'm aware of it all the time, because it seems to strike when I'm out."

- The definition of fecal incontinence treatment success :

- Focused on improvements to quality of life

- Only one patient mentioned a numerical reduction of incontinence episodes.

- Success was often defined by patients as restoration of their self-confidence.

"It has to be tailor[ed] based on individual sort of expectations and ultimately, comes down to quality of life."

\section{Discussion:}

- Access to effective treatments is a real barrier for Canadians suffering with fecal incontinence.

- Programs to treat Fl should focus on improvement of overall quality of life rather than a reduction of fecal incontinence episodes.

- Our findings can be compared to the existing literature on the outcomes of fecal incontinence. There is no standardized accepted definition of success when treating patient with fecal incontinence.

- Our findings support the use of instruments such as FISI and FIQL as they both include markers of quality of life.

- This study also suggests that care of incontinent patients could be improved by implementing educational initiatives both for patients and healthcare providers.

- This study is limited by the small numbers of interviews conducted and the lack of patients not seeking medical help in our sample.

\section{The Ottawa | L'Hôpital Hospital d'Ottawa}

